\title{
New development in relay protection for smart grid
}

\author{
Baohui Zhang ${ }^{1^{*}}$, Zhiguo $\mathrm{Hao}^{1}$ and Zhiqian $\mathrm{Bo}^{2}$
}

\begin{abstract}
This series of papers report on relay protection strategies that satisfy the demands of a strong smart grid. These strategies include ultra-high-speed transient-based fault discrimination, new co-ordination principles of main and back-up protection to suit the diversification of the power network, optimal co-ordination between relay protection and auto-reclosure to enhance robustness of the power network. There are also new development in protection early warning and tripping functions of protection based on wide area information.

In this paper the principles, algorithms and techniques of single-ended, transient-based and ultra-high-speed protection for EHV transmission lines, buses, DC transmission lines and faulty line selection for non-solid earthed networks are presented. Tests show that the methods presented can determine fault characteristics with ultrahigh-speed $(5 \mathrm{~ms}$ ) and that the new principles of fault discrimination can satisfy the demand of EHV systems within a smart grid.
\end{abstract}

Keywords: Smart grid, Fault transient component, Ultra-high-speed protection

\section{Introduction}

Relay protection is the key to the safe operation of a power system. The functions of relay protection have been developed along with enhancements to electrical power systems and the implementation techniques developed with the related areas of science and technology. Ensuring the function of a relay to satisfy the requirements of the development of the smart grid and perform the protection task with high reliability, involves a series of key technical issues. These issues, for example, include among others, the principles, criteria and algorithms for discriminate internal and external faults. In order to remove the faulty component with minimum area of interruption, it requires the setup of multiple protection relays and their technical coordination. In order to meet the above requirements, protection devices based on various hardware platforms, different techniques and the on site operational management of these devices are adopted. Each stage is instrumental in ensuring that the relay operates correctly. This paper mainly outlines the research and development undertaken for the protection

\footnotetext{
* Correspondence: bhzhang@mail.xjtu.edu.cn

${ }^{1}$ School of Electrical Engineering, Xi'an Jiaotong University, Xi'an 710049,

China

Full list of author information is available at the end of the article
}

system based on the latest digital protection technology and these developments will greatly influence the type of relays produced in the future.

China is building a strong smart grid and is constructing a 750/1000 kV transmission network to provide super power transmission capability. To utilize this kind of transmitting capacity considering the transient stability limits, it relies on the performance of the relay protection. Therefore, the research and development of ultra fast protection with response speed within $5 \mathrm{~ms}$ has great practical significance. With the development of high speed Digital Signal Processor (DSP) embedded system techniques and the application of optical sensor, the latest protection hardware posses the ability to record and compute the detailed fault transients. The mathematical tool Wavelet transform used to analyze the characteristics of the non-periodic sudden changes of a signal provides a powerful tool for the analysis and the computation of fault transient data. The fault transient components consist of information including the fault location and type. The above mentioned device and algorithms can be used to develop ultra high speed protection relays to identify the fault location and fault type based on detecting and processing the high frequency components of such fault transients. 
For over a century, many protection principles have been developed based on the lumped parameter model and utilizing the differences between the power frequency voltage and current during the normal operation and fault states. The vector and symmetrical component analysis methods have also been developed to constitute the theoretical basis for fault identification. In order to eliminate the non-power frequency components of the fault transient period, many filtering methods have been developed and have become an important component of the protection algorithms. The maturity of these theories and algorithms over the years enables many power system fault conditions to be correctly and selectively removed very quickly. However, to obtain complete information over a power frequency period $(50 \mathrm{~Hz})$ requires at least $20 \mathrm{~ms}$ to collect. To remove a fault faster, an approximation technique is adopted that uses the information from $1 / 2$ or $1 / 4$ of the period to represent the whole period to reduce the operational zone of the protection but still guarantee the selectivity.

In the 1960's, people started to develop protection using the fault generated transient wave front, which marked the start of using fault transients for protection. Due to the limitation of the available technical means and the complexity in identifying the reflections of the wave front from the fault point, the prototype relays developed at that time do not offer today's desired reliability and speed. However, site records indicate that the fault transients contain an abundant amount of information about the fault location and the type of fault in the fault record. Through the examination and analysis of these characteristics, new protection principles can be developed to solve the problems which can not be resolved by power frequency based protection principles. This approach can also further increase the response speed of the protection relay to meet the requirements of the UHV/EHV power system, which is in line with the development trends of the technology and today's power systems.

Although the concept of the smart grid was only raised recently, researchers have already undertaken research into many of the aspects needed to meet the development requirements of the latest power grid technology. This paper will introduce the research work over the past 10 years conducted by the authors' team on: the new ultra high speed protection principle, device implementation and test results obtained by utilizing the transient characteristics of the AC and DC transmission systems. Due to the space limitation, this paper will concentrate on the research results, while the detailed principles, algorithms and development process of the techniques can be found in the references.

\section{Transient based protection for ultra high voltage transmission lines}

The protection diagram is shown in Fig. 1 Discriminating the internal and external faults is achieved by utilizing the differences in the magnitude and direction of the high frequency voltages and currents.

\section{Main protection component and the principle employed [1-12]}

(1) Starting Unit(SU): based on the sudden change in the characteristic (Lipschitz coefficient) of the initial traveling wave caused by a fault on the transmission line, it adopts the "wavelet modular change and

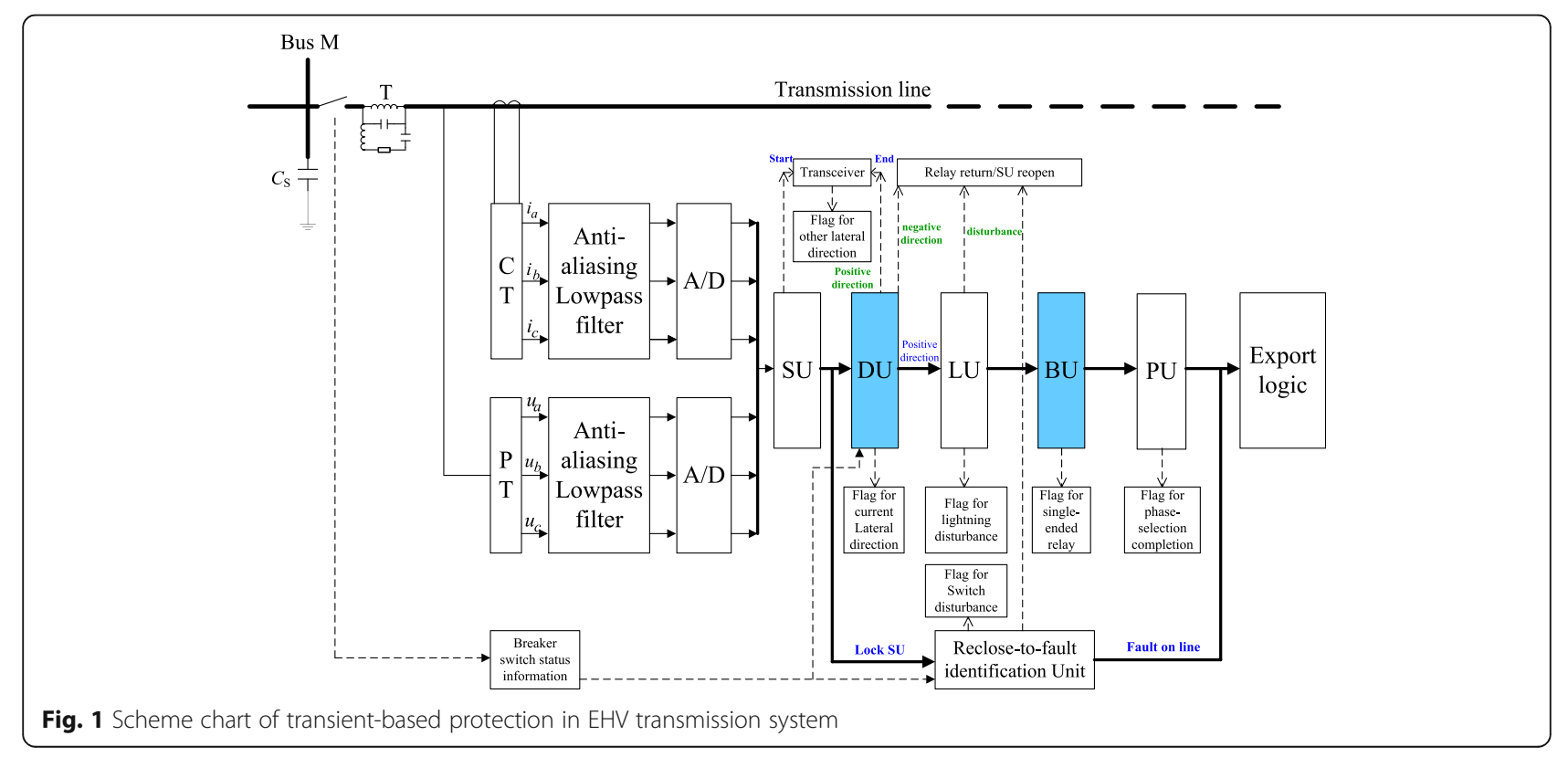


Wavelet Transform Module Sum" method to detect the signal singularity and to construct the fault start and interference not start element.

(2)Direction Unit(DU): when the fault is from the forward direction, the ratio of the positive direction traveling wave to the reverse direction traveling wave is $\Delta \mathrm{uf} / \Delta \mathrm{ub}=\mathrm{kr}$, where $\mathrm{kr}$ is the reflection coefficient and $0<|\mathrm{kr}|<1$. For the reverse direction fault, $\Delta \mathrm{uf} / \Delta \mathrm{ub} \rightarrow \infty$. Based on this the principle and algorithm for identification of fault direction can be constructed.

(3)Boundary Unit: the protection boundary is composed of the line trap and the equivalent grounding capacitance of busbar. The magnitude of the high frequency signal for an internal fault is significantly higher than that of an external one (for an external fault, the high frequency signal has difficulty passing through the boundary). Utilizing the magnitude ratio of the high to low frequencies of the reverse voltage or current traveling waves, the internal and external fault can be distinguished.

(4)Lightning Strike Identification Unit(LU): the algorithm extracts and computes the spectral energies of the $0-5 \mathrm{kHz}$ and $5-10 \mathrm{kHz}$ signals. The ratio of the spectral energies in each of two frequency bands is used to distinguish between a normal fault and a lightning strike. A higher ratio indicates a fault, a lower ratio a lightning strike.

(5) Reclose-to-fault identification Unit: there are significant differences in the time intervals of the initial traveling wave and the reflected one, the signal polarity and the frequency domain characteristics of the current for a faulty line and a healthy line. These differences in such characteristics can be used to develop the principles and algorithms for this element.

(6) Phase selection Unit(PU): the phase selection unit can be categorized into two types:1) traveling wave phase selection method, which utilizes the magnitude and polarity of the traveling wave in the current waveform; 2) the transient energy based phase selection method, which measures the relative magnitudes of the transient energies of the three phase current and determines the faulted phase based on the coupling relationship between the faulted and the un-faulty phases.

\section{Experimental device used for the transient based protection}

\section{$[3,4,13]$}

There are two devices. One of them is the prototype relay for transient based protection, which is able to simultaneously sample 8 channels of transient signal with $400 \mathrm{kHz}$ sampling rate and $12 \mathrm{bit} \mathrm{A} / \mathrm{D}$. The other one is the analogue transient signal generator, which is able to rapidly convert the digital data obtained from on site fault recorders or EMTP simulations into analogue signals. Based on over 800 groups of simulation data obtained from EMTP, 4000 real-time tests were conducted. The entire protection algorithms include 34728 multiplier and 31785 additional operations. Tests show that this prototype relay is able to make correct decisions within $4 \mathrm{~ms}$ for all cases.

\section{Discussions on relevant issues of transient based protection}

\section{(1)Instrument transformer [14]:}

Tests were conducted for electromagnetic types of instrument transformers. The high frequency signals are transferred through the windings turn to turn transformer action and the turn to earth capacitances of the primary and secondary windings. Tests show that the voltage transformer is able to transfer high frequency signals under $700 \mathrm{kHz}$ and the current transformer under $400 \mathrm{kHz}$. The cut-off frequency is related to the method of construction of the instrument transformer regardless whether the transformer has an iron core or not.

(2) The influence of the switching operation and the high frequency power line carrier signal [15].

Extensive simulation tests show that there is no maloperation for switching operations on both the protected and neighboring lines. The energy in the power line carrier signal is significantly lower than that in the generated fault signal in the high frequency band of interest. When both signals exist in the system, the sensitivity of the fault discrimination for the boundary element will be slightly lower, but a correct decision can still be made for the external fault.

\section{Studies on the whole hvdc transmission line ultra high speed protection based on single end measurement [16-18]}

The connection diagram of the HVDC system is shown in Fig. 2. Within the dashed line is the HVDC transmission line. The DC filtering group and the smoothing reactors are installed at the two ends of the lines, which provide a natural boundary for the high frequency transients. For the internal and external fault with respect to the location at the dashed line where the protection relays are installed, there are distinguishing differences in the high frequency voltage and current signals. The single end ultra high speed protection for DC line in this situation is more reliable than that for $\mathrm{AC}$ lines and is not influenced by the fault instance. 


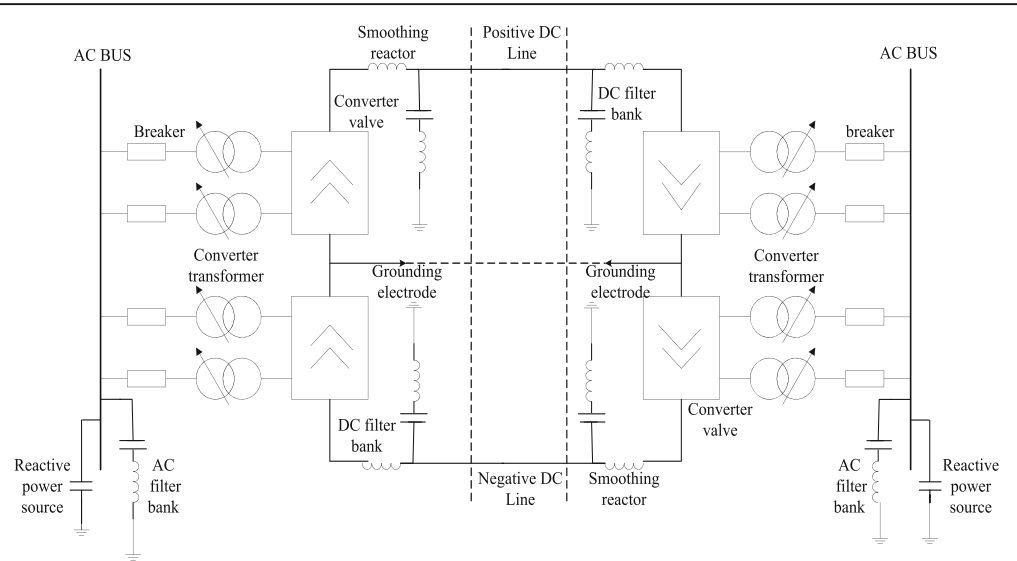

Fig. 2 Schematic diagram of HVDC transmission system

At present DC line protection is mainly provided by manufacturers e.g. $\mathrm{ABB}$ and Siemens. The discrimination criterion of the single end protection algorithm is based on the super-imposed voltage, the rate of change of the voltage traveling wave, and the gradient of the change in the current. On 27th August 2007, the voltage rate of change of the traveling wave protection did not operate for a high resistance fault (fault resistance 295 $\Omega$ ) on the GeNan HVDC transmission line. Similarly, during the operations of the TianGuang HVDC transmission line project in recent years, the voltage rate of change of the traveling wave protection has often failed to operate for high impedance faults. Since the rate of change protection is affected by fault location and fault resistance, its operation threshold is set to ensure no mal-operation for external fault. As a result of this it can not guarantee the reliable operation for internal fault.

The principle diagram of the single ended ultra high speed protection principle, utilizing the characteristics of the high frequency signal for a DC transmission line is shown in Fig. 3. From Fig. 3 it follows that:

(1) The principle and algorithm of the start element: First it detects the change of current and adopts the improved gradient algorithm. Using the sum of the current 3 samples and subtracting this sum from the last 3 samples, protection will start when the change of the current gradient exceeds a certain value. The calculation is simple but provides high sensitivity for high resistance fault.

(2) Principle and algorithm of boundary element: The characteristic of the boundary frequency for a DC transmission line is shown in Fig. 4. This figure shows that the magnitude of the high frequency signal for the detected internal faults is 50db higher than that for external faults. This difference can be used to distinguish between internal and external faults. Using the db3 wavelet transform to extract the fault components $\Delta \mathrm{u}$ and $\Delta \mathrm{i}$ of the voltage and current, the high frequency forward voltage $\Delta \mathrm{u}_{\mathrm{f}}$ and reverse voltage $\Delta \mathrm{ub}$ of the traveling wave are computed. In this way the energies of the high frequency fault components can be used to establish the protection criteria to distinguish between internal and external faults.

(3) Fault polarity detection: The two pole HVDC transmission line is a double-circuit construction. Due to the coupling between the two lines, a fault on one pole will induce relatively high electromagnetic transients of voltage and current in the healthy line. Therefore, it is difficult to identify the faulty line based on the measurement of the magnitude of the high frequency signal at one end. When comparing the signals on the two lines with a low frequency range,

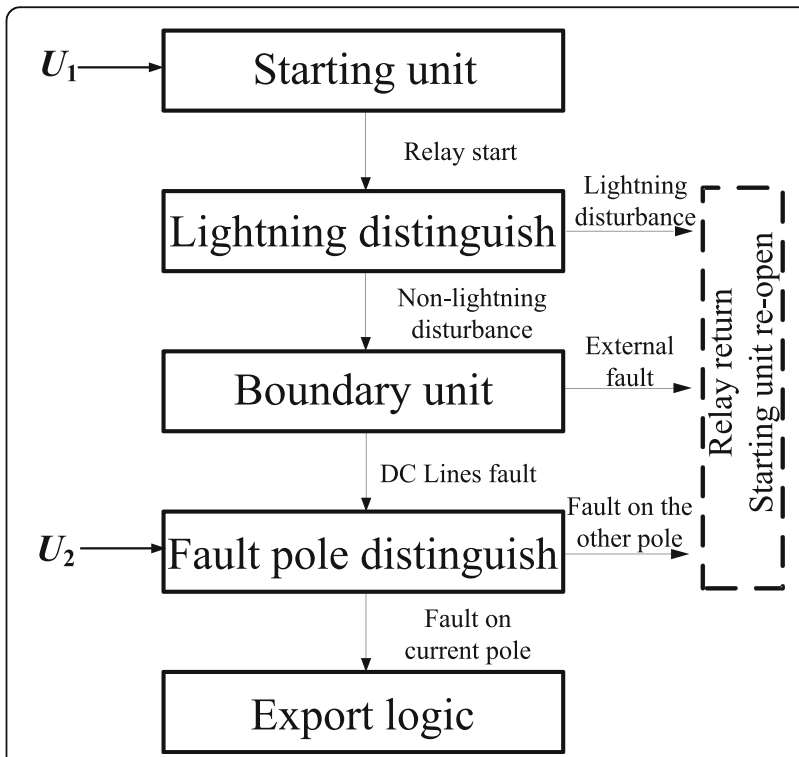

Fig. 3 Diagram of single-ended transient-based protection for HVDC transmission lines 


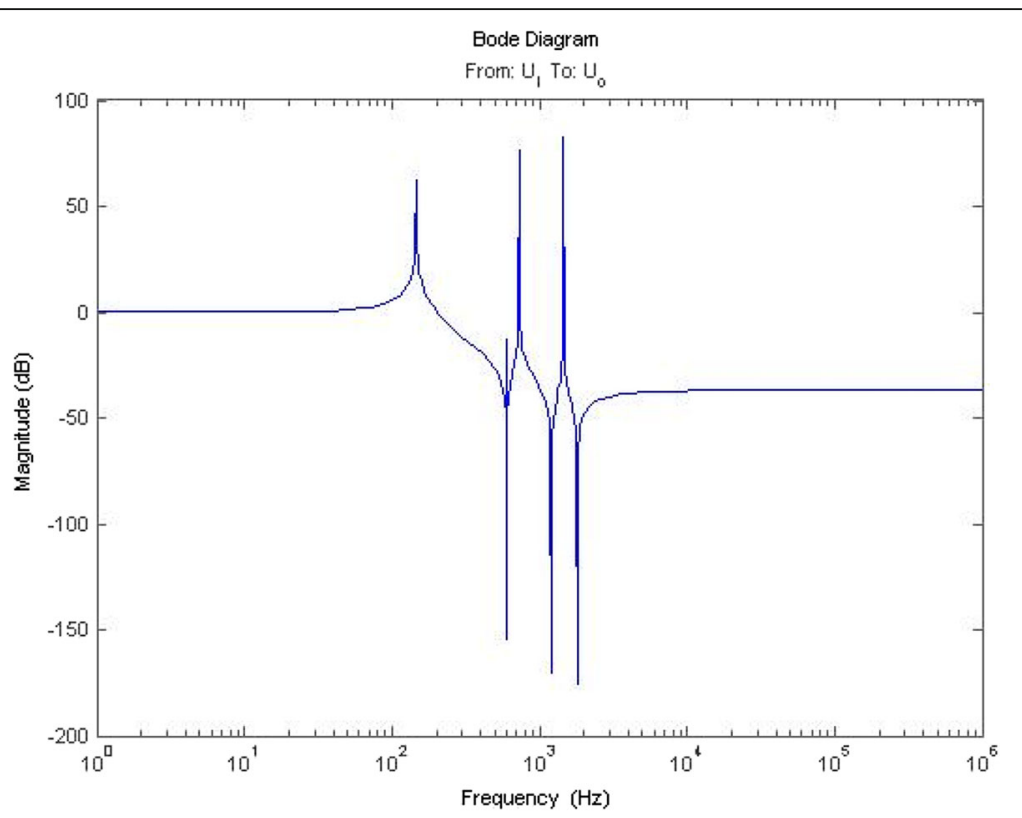

Fig. 4 Amplitude-frequency characteristic of the DC line boundary $\left(10^{\circ} \sim 10^{6} \mathrm{~Hz}\right)$

the signal of the faulty phase is higher than that of the healthy ones. This criterion can be used to distinguish whether a fault is on its protected line.

Simulation tests of the prototype relay: A typical $1043 \mathrm{~km}$ long, $\pm 500 \mathrm{kV}$ HVDC transmission system is simulated using the EMTP software. The sampling frequency is $100 \mathrm{kHz}$ and the $\mathrm{db} 3$ wavelet transform is used. The entire protection algorithm requires 5760 multiplier and 4800 summation calculations and can be computed within $300 \mu$ s using C32 series of DSP. By taking $1 \mathrm{~ms}$ as the data window, the protection can be easily computed within $2 \mathrm{~ms}$. Simulation tests were conducted for over one thousand fault cases in the test system. The protection remained stable for all cases. For a lightning strike on the DC lines and $500 \Omega$ fault resistance, the protection is able to provide high sensitivity with an operation threshold 10 times higher than the setting threshold. However, the protection algorithm based on the rate change of voltage $\mathrm{du} / \mathrm{dt}$ can not distinguish between internal and external faults when the fault resistance is over $300 \Omega$.

\section{Ultra high speed busbar protection $[19,20]$}

The PSCAD/EMTDC software was used to assess the performance of the transient based busbar protection. The equivalent busbar model, where all the busbar stray capacitances are represented by one capacitor, can not truly represent the transient process for busbar internal fault. For this situation the correct models, which take into consideration of the stray capacitance in various locations for the transformer, arrester, CVT and line trap should be used. The typical $3 / 2$ connection diagram of the simulation is shown in Fig. 5.

The sampling frequency is $400 \mathrm{~Hz} / \mathrm{s}$ and the 4 order Bspline derivative function is used as the foundation dyadic wavelet transform. The performances of the three algorithms were examined to compare the polarity of the traveling wave current, the differential traveling wave and the traveling wave energy for the comparison of the busbar protection. Two out of three modes should meet the criteria when 3 modes are used. Simulation results show that all 3 principles for busbar protection can distinguish between internal and external faults within $2 \mathrm{~ms}$ and the traveling wave differential protection possesses the highest sensitivity and reliability. The algorithm is also less influenced by any branch traveling wave current due to the transformer, which can result in difficulties when determining the polarity and direction of the traveling wave current. The reliability, therefore, of the algorithm requires further research. The main advantages of the algorithm are the fast speed, and the fact that it is not influenced by CT saturation and fault path resistance.

\section{Faulted line selection device for non-effective earth systems [21-23]}

For many years, stable state quantities have been used for line selection for non-effective earthed systems. However, the magnitudes of the stable state quantities are small, and can become even smaller when taking into consideration of the compensation effect of the Petersen coil, the fault path resistance and any CT errors. As a result, the success rate for this type of faulty line selection device is very low, especially when large fault 


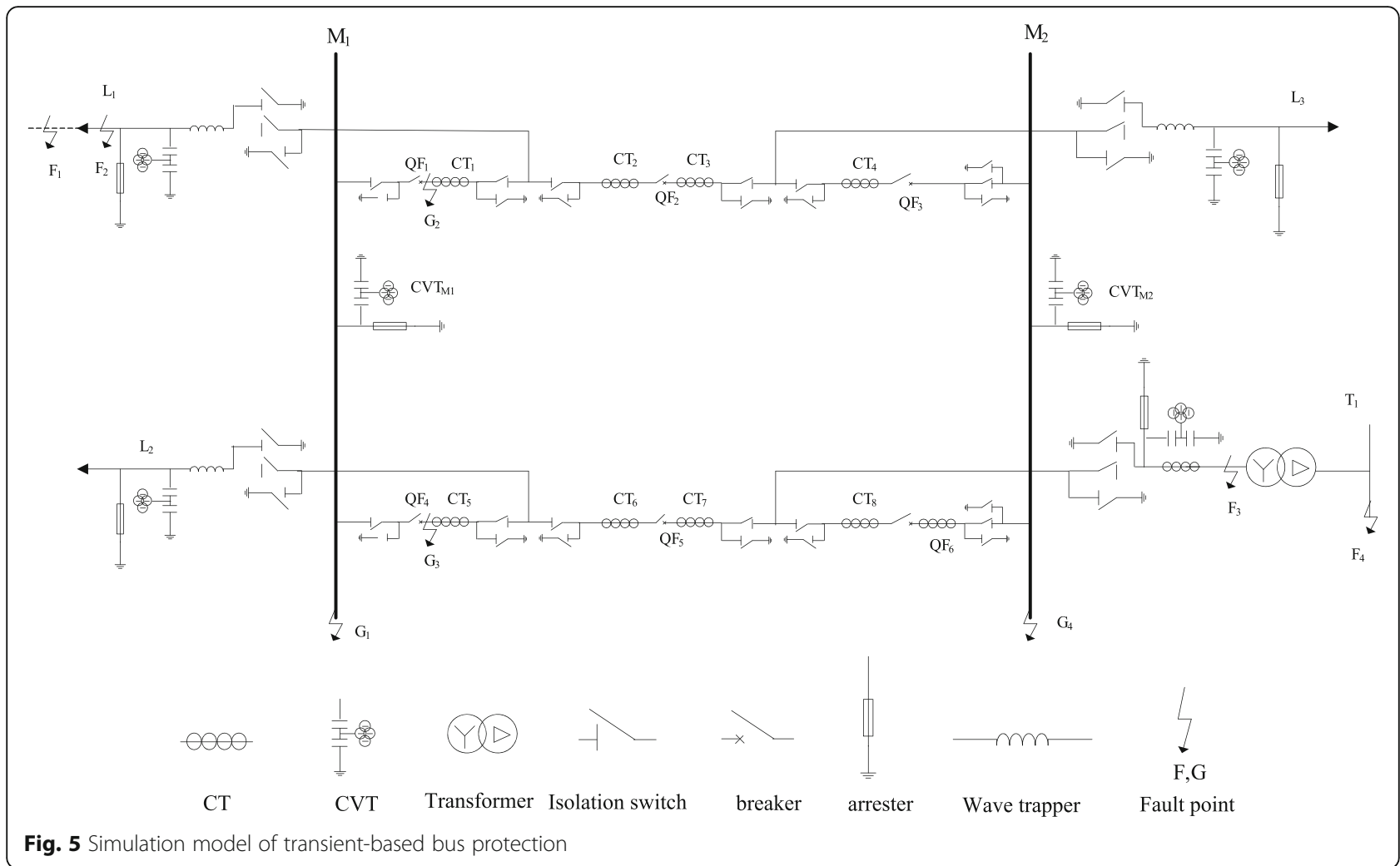

resistance and arcing fault are involved. Although there are many installations on-site, power engineers have generally lost faith in this type of device due to its low accuracy.

The magnitude of the transient capacitance current can reach up to 10 times or even higher of the stable state capacitance current without the effect of a Petersen coil. Figure 6 compares the direction and magnitude of the zero sequence transient currents for every feeder.
The current in each feeder follows into the busbar and the one with the highest magnitude is from the faulty feeder. However, the above mentioned characteristics are only true for a particular frequency band, and this band varies and is also influenced by network parameters and fault modes etc. Therefore, adaptively selecting the frequency band is the key for accurate and reliable faulty line selection.

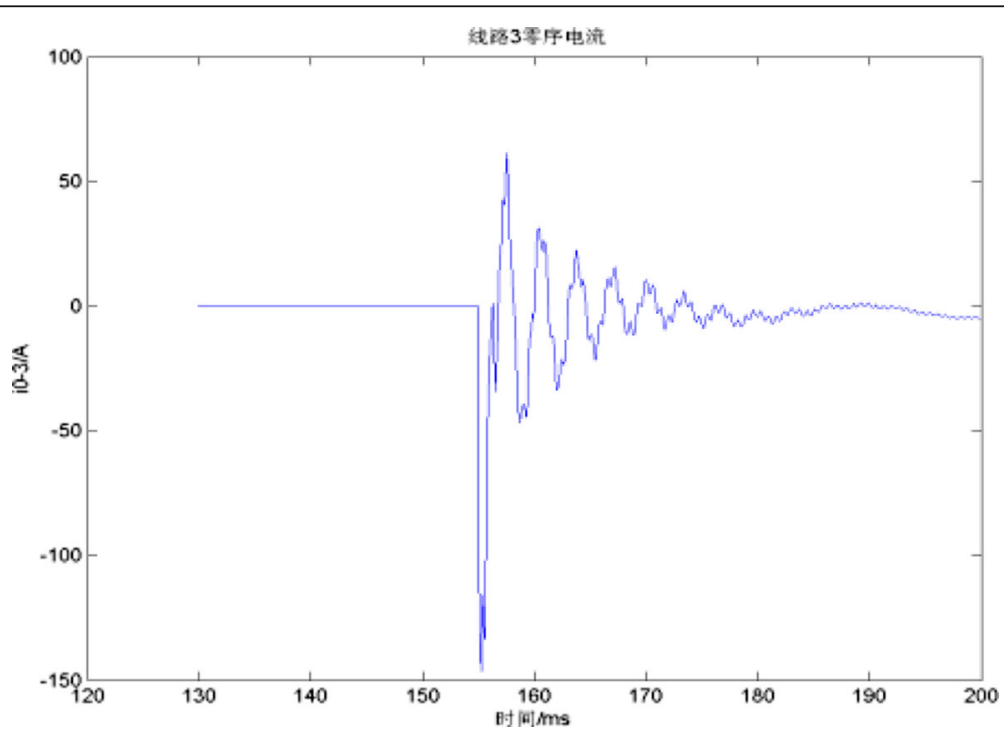

Fig. 6 Zero-sequence current of fault line (the shortest line) 


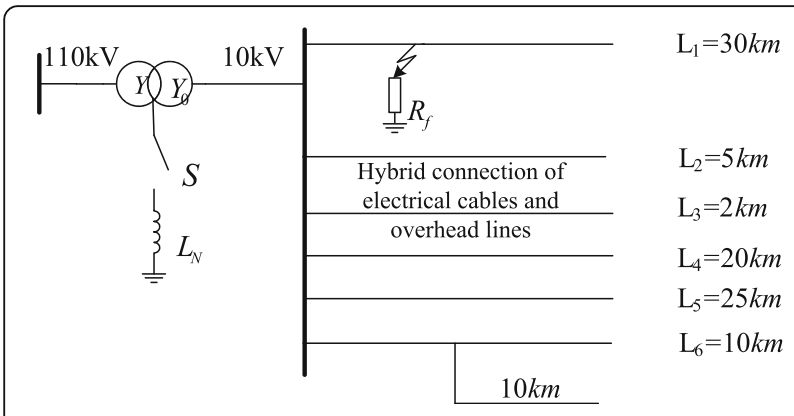

Fig. 7 Diagram of non-solid earthed distribution system

For the mixture overhead line and underground cable system shown in Fig. 7, RTDS simulation was conduced for over 10 thousand cases for unearthed, neutral connection and earth connection through a Petersen coil. This was done for under and over compensation and the accuracy was found to be over $97 \%$. There were no wrong line selection record for a number of devices installed on-site either.

\section{Conclusion}

The construction of the strong smart grid gives rise to demanding requirement for the protection relay in respect to both speed of operation and reliability. With the development of high speed DSP and the application of optical sensor, the utilization of fault transient information for fault identification is no longer a difficult task. The implementation of protection principles based on transient information has already become possible.

Due to the inconsistent nature of the wave impedance for various power apparatuses and the reflection and refraction characteristics of their interconnection points, the fault transients contain abundant information about the fault location and type. By correct analysis and the full utilization of such information it is possible to construct ultra high speed and highly sensitive AC, DC and busbar main protection. In addition faulty line selection for neutral non-effective earth systems using transient characteristics offers high sensitivity and reliability that is not influenced by the ways of neutral compensation.

\section{Authors' contributions}

$\mathrm{BHZ}$ investigated the framework of relative relay protection for smart grid, and drafted the manuscript. ZGH summarized the history and recent development of smart grid relay protection. ZQB participated in typesetting and revision of the manuscript. All authors read and approved the final manuscript.

\section{Competing interests}

The authors declare that they have no competing interests.

\section{Author details}

'School of Electrical Engineering, Xi'an Jiaotong University, Xi'an 710049, China. ${ }^{2}$ XUJ Group Corporation, Xuchang 461000, China.

\section{References}

1. Ha, H.X. (2002). The study of boundary protection for EHV transmission lines. Thesis: Xi'an Jiaotong University.

2. Duan, J.D. (2005). Study of ultra-high-speed transient- based protection for extra-high-voltage transmission system. Thesis: Xi'an Jiaotong University.

3. Zhang, S. X. (2004). Research on principle simulation platform of transmission line single-ended transient based protection. Xi'an: Xi'an Jiaotong University.

4. Zhou Yi. (2006). Research on implemention technology of utra-high-speed transient-based protection for transmission line. Thesis: Xi'an Jiaotong University.

5. Duan, J. D., Zhang, B. H., \& Zhou, Y. (2005). Study of ultra-high-speed transient-based directional relay. Proceedings of the CSEE, 25(4), 7-12.

6. Duan, J. D., Zhang, B. H., Li, P., et al. (2007). Single-ended transient-based protection for EHV transmission lines: principle and algorithm. Proceedings of the CSEE, 27(3), 45-51.

7. Duan, J. D., \& Zhang, B. H. (2004). Study of starting algorithm using traveling-waves. Proceedings of the CSEE, 24(9), 30-36.

8. Duan, J. D., Ren, J. F., Zhang, B. H., et al. (2006). Study of transient approach of discriminating lightning disturbance in ultra-high-speed protection. Proceedings of the CSEE, 26(23), 7-13.

9. Duan, J. D., Zhang, B. H., Ren, J. F., et al. (2007). Single-ended transientbased protection for EHV transmission lines: basic theory. Proceedings of the CSEE, 27(1), 38-43.

10. Duan, J. D., Luo, S. B., Zhang, B. H., et al. (2007). Study of discriminating approach for switching into fault line in ultra-high-speed protection. Proceedings of the CSEE, 27(10), 78-84.

11. Duan, J. D., Zhang, B. H., \& Zhou, Y. (2005). Study of fault-type identification using current traveling waves in extra-high-voltage transmission lines. Proceedings of the CSEE, 25(7), 58-63.

12. Duan, J. D., Zhang, B. H., Zhou, Y., et al. (2006). Transient-based faulty phase selection in EHV transmission lines. Proceedings of the CSEE, 26(3), 1-6.

13. Zhang, S. X., Zhang, B. H., \& Duan, J. D. (2004). Research on development platform for transient-based protection. Relay, 32(15), 34-38.

14. Yin, X.C. (2006) Testing and simulation on secondary electromagnetic transformer for high frequency character. Thesis: Xi'an Jiaotong University.

15. Xue, J., Zhao, J. Q., Zhang, B. H., et al. (2007). Research on anti-interfering capability of ultra-high-speed transient- based Protection for transmission line. High Voltage Apparatus, 43(5), 374-377.

16. Cao, R.F. (2006). Study of Non-unit transient-based protection for HVDC transmission line. Thesis: Xi'an Jiaotong University.

17. Cao, R. F., You, M., Zhang, B. H., et al. (2009). Ultra-high-speed transient-based protection for HVDC lines. Beijing: 2009 International Conference on UHV Transmission.

18. You, M., Zhang, B. H., Cao, R. F., et al. (2009). Study of non-unit transient-based protection for HVDC transmission lines. Wuhan: Asia-Pacific Power and Energy Engineering Conference.

19. Chen, J. (2004). Simulation of protection principle of the busbar protection, by used of fault transient. Thesis: Xi'an Jiaotong University.

20. Duan, J. D., Zhang, B. H., \& Chen, J. (2004). Study on the current traveling- wave differential bus protection. Automation of Electric Power Systems, 28(9), 43-48.

21. Zhao, H. M., Zhang, B. H., Duan, J. D., et al. (2006). A new scheme of faulty line selection with adaptively capturing the feature band for power distribution networks. Proceedings of the CSEE, 26(2), 41-46.

22. Zhang, B. H., Zhao, H. M., Zhang, W. H., et al. (2008). Faulty line selection by comparing the amplitudes of transient zero sequence current in the special frequency band for power distribution networks. Power System Protection and Control, 36(13), 5-11.

23. Xu, J. D., Zhang, B. H., You, M., et al. (2009). Fault line selection device for non-solid earthed network based on transient zero-sequence current features. Electric Power Automation Equipment, 29(4), 101-105. 